\title{
Full field investigation of salt deformation at room temperature: coopera- tion of crystal plasticity and grain sliding
}

\author{
M. Bourcier, A. Dimanov, E. Héripré \& J.L. Raphanel \\ LMS,UMR 7649, Ecole Polytechnique, Palaiseau, France. \\ M. Bornert \\ Laboratoire Navier, UMR 8205, Ecole des Ponts ParisTech, Marne La Vallée, France. \\ G. Desbois \\ Structural geology, Tectonics and Geomechanics, RWTH Aachen University, Germany.
}

\begin{abstract}
We observed with optical and scanning electron microscopy halite samples during uniaxial compression. Surface displacement fields were retrieved from digital images taken at different loading stages thanks to digital image correlation (DIC) techniques, on the basis of which we could 1) compute global and local strain fields, 2) identify two co-operational deformation mechanisms. The latter were 1) crystal slip plasticity (CSP), as evidenced by the occurrence of slip lines and computed discrete intracrystalline slip bands at the grain surfaces, 2) interfacial micro-cracking and grain boundary sliding (GBS), as evidenced by the computed relative interfacial displacements. The heterogeneities of the strain fields at the aggregate and at the grain scale, and the local contributions of each mechanism were clearly related to the microstructure, i.e. the relative crystallographic orientations of neighboring grains and the interfacial orientations with respect to the principal stress.
\end{abstract}

\section{INTRODUCTION}

Halite has been extensively studied both as a rock forming mineral with industrial applications (storage of wastes and hydrocarbons in mines and deep caverns), and as an analog viscoplastic material for the purpose of academic investigations of plasticity, texture development and some more specific mechanisms such as pressure solution (Urai et al., 1987; Wenk et al., 1989; Hickman and Evans, 1995; Peach and Spiers, 1996; Martin et al., 1999; Lebensohn et al., 2003; Liu et al., 2005; Wenk et al., 2009). There is also a renewed interest in studies of the mechanical behavior of halite under low stresses (Bérest et al., 2005) and cyclic loading, owing to a new projected use of salt cavities as storage for compressed air, which is in turn an alternative for storage of excess "green energy", produced by renewable but fluctuating sources (solar, Aeolian,...). In this work, we present results of a multi-scale micromechanical approach, aimed at the identification of the physical mechanisms by which halite deforms plastically at room temperature.

\section{EXPERIMENTS}

\subsection{Sample preparation}

A sound micromechanical investigation relies on the strict control of sample microstructure. Therefore we synthesized a damage-free material, characterized by narrow grain size distribution, low porosity and limited deformation related microstructures. We followed similar techniques as those developed by Peach and Spiers (1996).

The material was obtained by pressing of pure laboratory salt powder (99,9 \%). First, the powder was cold pressed during 48 hours in an oedometric cell in order to reduce the porosity. In a second time, the cell is externally heated in order achieve hot pressing at $100 \mathrm{MPa}$ and $150^{\circ} \mathrm{C}$ during one week. The resulting cylindrical blocks of about 10 $\mathrm{cm}$ in height and $8 \mathrm{~cm}$ in diameter (fig 1a) present very good cohesion, allowing for specimen coring and machining. Observed by SEM (scanning electron microscopy), after classical polishing and wet cleaning, the "as-hot-pressed" material showed a wide grain size distribution $(20 \mu \mathrm{m}-400 \mu \mathrm{m})$ and numerous wavy interfaces, which indicated a nonequilibrium state (Fig. 1b). We also observed sample surfaces prepared by BIB (broad ion beam), which is an atomic scale erosion process based on ion beam sputtering (Ar source), allowing to prepare 2D flat undamaged surfaces (curtaining less than $5 \mathrm{~nm}$ deep) up to $2 \mathrm{~mm}^{2}$, suitable for high-resolution SEM imaging of sub-micrometric microstructures (Desbois et al., 2011). This technique revealed numerous inherited sub-grain boundaries, related to crystal plasticity recovery during the hot-pressing stage (Fig. 2a), and also sub-micrometric interfacial porosity $(<2$ vol. $\%)$, which is impossible to preserve during classical sample preparation.

In order to obtain a more homogeneous and equilibrated microstructure, we further annealed the samples at $750^{\circ} \mathrm{C}$ (at atmospheric pressure) during 
48 hours. The resulting microstructure presents flat or slightly curved interfaces and narrow grain size distribution, ranging from $250 \mu \mathrm{m}$ to $500 \mu \mathrm{m}$ (fig 1b). The sub-grain boundaries vanished during the grain growth process. But, the involved interfacial migration also resulted in porosity coalescence (Fig. 2 ), and even in porosity increase (up to $3 \%$ ).
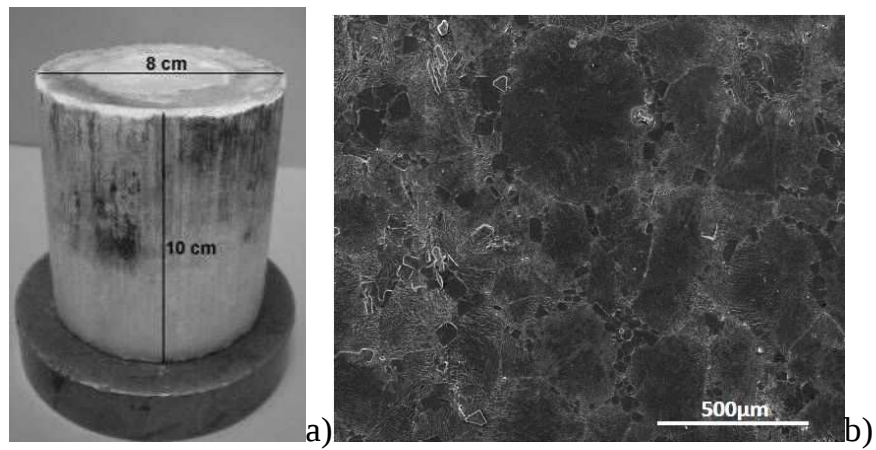

Figure 1 : a) Hot-pressed block. b) Microstructure of hotpressed synthetic salt.

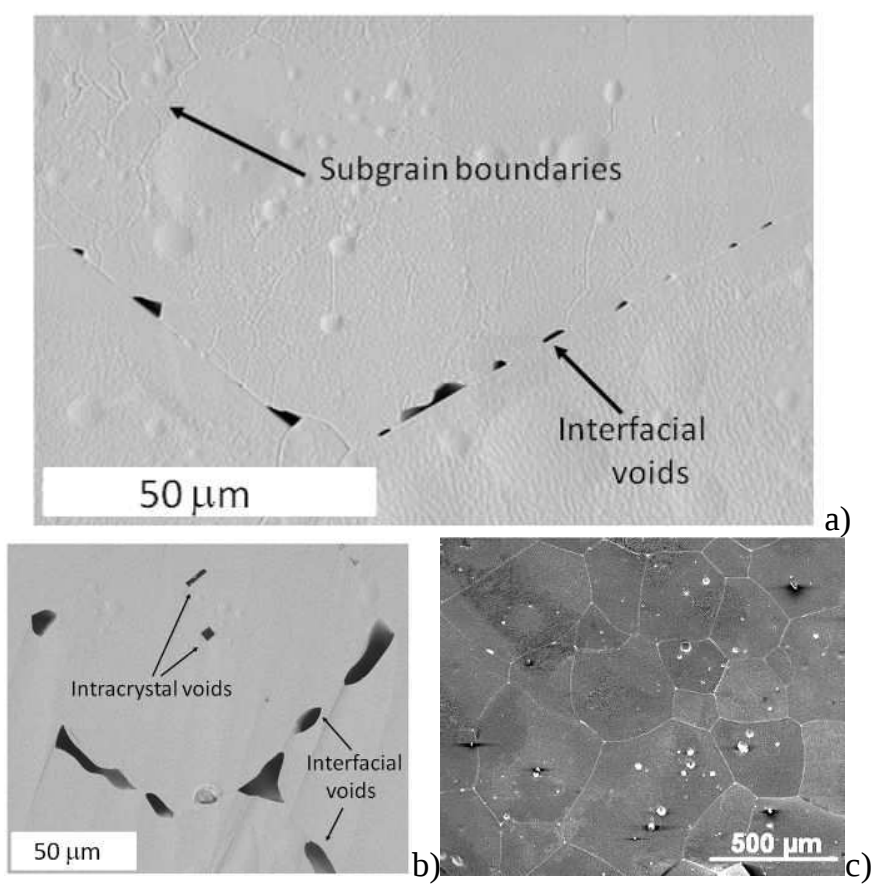

Figure 2 : SEM micrographs. a) Detail of as hot-pressed sample (BIB preparation), with fine grains, subgrains and irregular interfacial micro-pores. b) Detail of annealed sample (BIB preparation), with larger grains, larger and smoothed interfacial pores, intracrystalline faceted pores (negative crystals). c) General microstructure of hot-pressed and annealed sample (classical preparation), with large, equiaxed and euhedral grains.

\subsection{Macroscopic compression tests}

Uniaxial compression of large $\mathrm{cm}$-sized samples was performed during observations by optical microscopy (OM) at the scale of the whole specimen. These so called "macroscopic tests" aimed at the full field mechanical characterization at a scale exceeding those of the representative volume element (RVE). For a polycrystalline material the RVE may be defined as the smallest volume of randomly oriented grains, which exhibits isotropic and homogeneous mechanical responses, whatever the anisotropy of the individual crystalline grains may be.

The uniaxial compression tests were realized with an electromechanical press with $100 \mathrm{kN}$ loading capacity. The cylindrical samples (c.a. $23.5 \mathrm{~mm}$ in height and $23 \mathrm{~mm}$ in diameter) were directly cored from the as-hot-pressed material. The sample surfaces were machined so that they were truncated along two diametrically opposite cords of $1.5 \mathrm{~cm}$ in length. The resulting two opposed and flat surfaces were carefully polished and paint sprayed, in order to offer surface markers, which are necessary for the full field measurements by digital image correlation (DIC, see next section). Both flat surfaces are observed by OM with two diametrically opposed digital cameras. One optical line allows for the observation of the whole specimen (16 Mpixel camera), whilst the other focuses onto a selected $4 \mathrm{~mm}^{2}$ sized area (4 Mpixel camera). The sample is loaded with a constant displacement rate of $1 \mu \mathrm{m} . \mathrm{s}^{-1}$, corresponding to a strain rate of $4 \times 10^{-5} \mathrm{~s}^{-1}$. Digital images are acquired each second. In order to minimize fretting (frictional effects) we placed Teflon spacers between the sample and the steel pistons.

We additionally performed conventional triaxial creep tests at room temperature on similar cylindrical (2.4 cm in diameter) synthetic salt samples, at constant differential stresses between 20 - 40 $\mathrm{MPa}$, and at $20 \mathrm{MPa}$ confining pressure (oil medium). Each sample was subjected to two duplicate series of five stress steps, reaching steady state strain rates. The experiments lasted between one to two months and the total shortening was of about $25 \%$.

\subsection{Microscopic compression tests}

Uniaxial compression of small mm-sized samples was performed in an environmental FEG (field emission gun) - SEM, which allowed the observation of the local behavior from the mesoscopic scale (or the aggregate scale, which is the one representative of the microstructure) to the grain scale. Typically, the aggregate scale represented several tens to hundreds of grains. At this scale, the interactions of numerous individual and anisotropic grains results in heterogeneous mechanical responses.

The SEM was fitted with a specifically designed miniaturized press (Fig. 3), equipped with a 5 $\mathrm{kN}$ load cell. Parallelepipedic samples (12 mm length, $6 \mathrm{~mm}$ side) were loaded at the constant displacement rate of $1 \mu \mathrm{m} . \mathrm{s}^{-1}$, corresponding to $10^{-4} \mathrm{~s}^{-1}$ strain rate. The surface markers were $\mu \mathrm{m}$-sized golden micro-balls obtained by high-temperature de-wetting of a thin gold film (see next section). Images are 
obtained in high vacuum mode with a size of $4096 \mathrm{x}$ 3775 pixels.
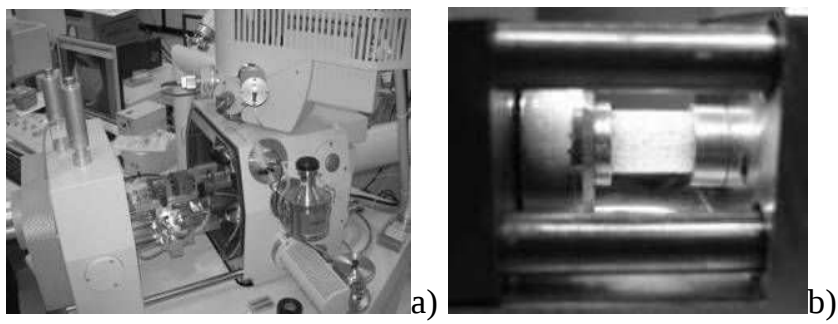

Figure 3 : a) SEM equipped with an uniaxial press. b) Synthetic salt sample (10 $\mathrm{mm}$ in length), horizontally fitted in the press.
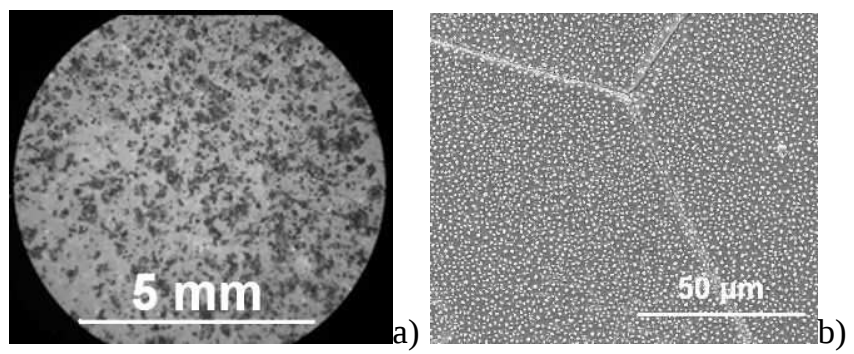

Figure 4 : a) Black paint spray. b) Micrometer-sized gold micro-droplets. The image shows a triple junction.

\subsection{Digital Image Correlation}

Digital Image Correlation (DIC) is an optical method for micro-extensometry allowing the calculation of the complete strain field at the surface of the loaded sample. We used the DIC software CorrelManuV (CMV), developed by $\mathrm{M}$. Bornert (Doumalin et al., 1999; 2003), which has recently been applied with success to "in-situ" SEM uniaxial compression of carbonates (Dautriat et al., 2011). DIC technique is based on the comparison and recognition of the grey scale levels of predefined correlation domains before and after loading. Unlike the shape of the correlation domain, the grey scale level is supposedly unchanged by the deformation process, which makes it possible to retrieve the displacement field and to calculate the corresponding strain field. The recognition of the correlation domains is realized by the minimization of a correlation criterion, which depends on the gray scale level. In practice, the accuracy of the method requires strong surface contrasts. A technique, to ensure a sufficient contrast consists in the deposition of surface markers. Actually, we have developed different techniques of marking, according to the studied scale. In Figure 4 we show black paint spray (Fig. 4a) and gold micro-balls (Fig. 4b) markers, which we use for macroscopic and microscopic scale investigations, respectively. The gold micro-balls are obtained as follows: 1) the sample surface is coated with a thin gold film, as usually done for metallization of insulators, 2) the sample is annealed at $500^{\circ} \mathrm{C}$, at which conditions the thin film retracts in the form of dendrites, followed by the formation of gold micro-balls, driven by surface energy minimization.

\section{RESULTS}

\subsection{Macroscopic tests}
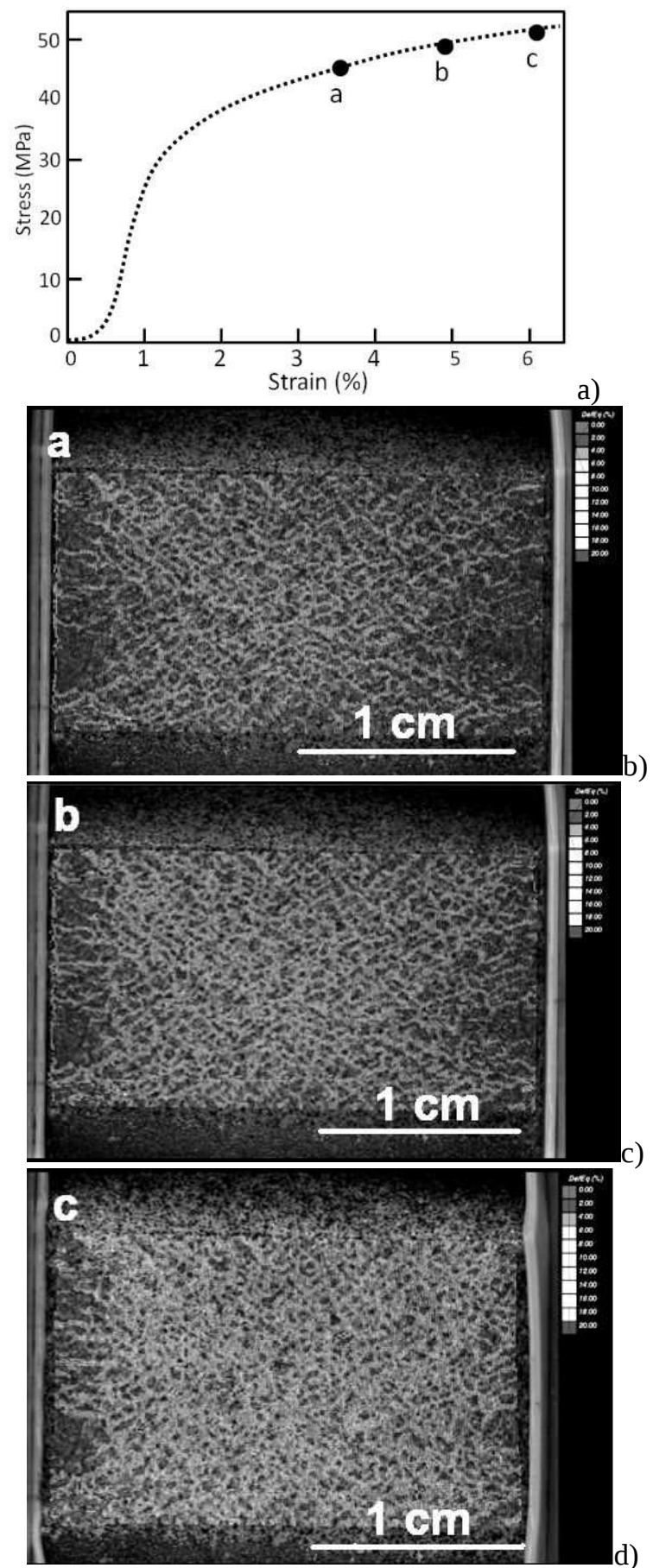

Figure 5 : Macroscopic stress - strain curve and macroscopic strain fields obtained by DIC at three consecutive steps of loading (measurement basis: $250 \mu \mathrm{m}$ ). Note in d) the pronounced indentation of the right hand Teflon spacer (light grey).

The results from one macroscopic test are summarized in Figure 5. The stress - strain curve, as recorded by the load cell and the piston displacement sensor (LVDT), is typical for a viscoplastic 
material characterized by moderate strain hardening. The whole specimen is presented in horizontal compression at three loading stages, which are indicated by $a, b$ and $c$ on both the images and the loading curve. DIC was realized with a correlation domain of $15 \times 15$ pixels and a pixel size equal to $7.4 \mu \mathrm{m}$. The von Mises equivalent strain, as computed by DIC along the central flat area, is superimposed onto the sample images. The evolution of the grey scale from dark to bright indicates the strain intensity. The measurement basis, which may be seen as the local optical (virtual) strain gage length, is chosen depending on the needed sensitivity in determining local heterogeneities. In the present case it was set to $250 \mu \mathrm{m}$, which corresponds to both the grain scale and the marker size.

End effects (fretting) were not avoided in spite of the Teflon spacers (in light grey), probably because those were indented (see Fig. $5 \mathrm{c}$ ). But, except in the vicinity of the pistons, the strain field clearly self-organizes into homogeneously distributed ductile shear bands oriented at about $45^{\circ}$ to the loading axis and presenting mm-sized interspacing. This latter internal length scale is in turn that of the aggregate (or the mesoscopic one), related to the polycrystalline microstructure. By the end of the experiment, we observed the classical development of conjugated diagonal macroscopic shear bands, which contained numerous tensile micro-cracks, aligned with the principle stress direction.

\subsection{Microscopic tests and mechanism identification}

We investigated two different scales in order to highlight the operational micro-physical mechanisms at a very local (grain) scale, and their interactions and self-organization of strain at the aggregate (mesoscopic) scale.

\subsubsection{Mesoscopic (aggregate) scale}

The combination of SEM observation and DIC allowed the identification of two mechanisms of plastic deformation. The slip lines emerging on the surface of nearly every grain of the sample (Fig. 6) result from the activation of crystal slip systems and testify of intracristalline plasticity as being the major deformation mechanism. Figure 7 presents the von Mises equivalent strain field given by DIC (correlation domain: 10x10 pixels, pixel size: $3 \mu \mathrm{m}$ ) at three different stages of loading, noted (a), (b) and (c) on the stress-strain curve (Fig. 7). The latter was computed on the basis of DIC performed for all of the 9 loading steps (data points). At the beginning, in stage (a) we can observe the development of discrete deformation bands within the individual grains, which correspond to slip bands due to crystal plasticity. With further loading, in stages (b) and (c) the deformation bands intensify within the individual grains. In addition, the grain-to-grain interactions and the influences of neighboring grains appear, as deformation bands develop at the aggregate scale, and propagate throughout the microstructure at nearly $45^{\circ}$ to the loading direction (Fig. 7 and Fig. 10).

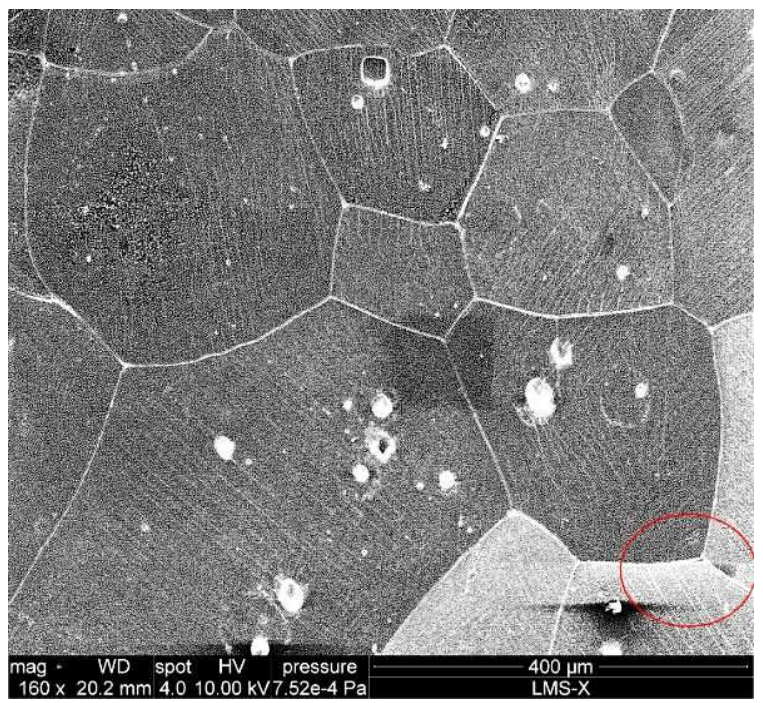

Figure 6 : SEM micrograph from the same sample and area as in Fig. 1b after deformation. Slip lines appear at the surface of the sample. The red circle indicates a triple junction where was detected grain boundary sliding accompanied by cavitation. The darker square area in the middle results from the contamination upon focused investigation of the triple junction (see Fig. 8).

The strain maps in Figures 7 and 10 also show that at the aggregate scale deformation bands alternate with areas of easier deformation (strain shadows). The latter are related to the presence of grains which are unfavorably oriented with respect to the principal stress, and whose slip systems are barely activated. The influence of such grains is not limited to the closest neighbors, but propagates across several grains throughout the microstructure, which actually highlights the mesoscopic length scale. These local interactions explain the self organization of alternating shear bands at larger scales (Fig. 5). In addition, DIC allowed the detection of strong localizations along some interfaces (Fig. 7), which actually corresponded to grain boundary microcracking and sliding (GBS). In some cases, GBS also resulted in cavitation (void opening) at triple junctions (Fig. 6). GBS and cavitation resulted in progressive accumulation of damage ultimately leading to failure. In the present case, according to the DIC strain analysis, the GBS mechanisms were responsible of about $8 \%$ of the total strain.

\subsubsection{Microscopic (grain) scale}



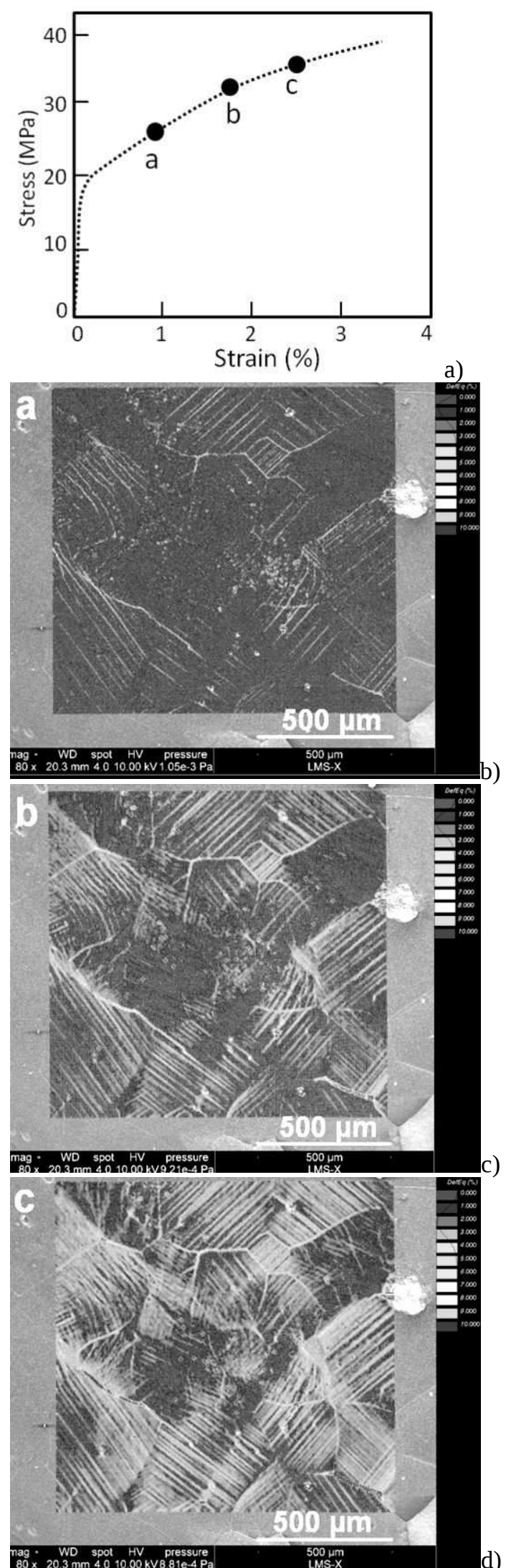

Figure $7:$ a) DIC computed stress and strain curve. b,c,d: Strain fields obtained by DIC at the aggregate scale at three consecutive steps of loading. Compression direction is horizontal. In b and c some interfaces show localization due to grain boundary sliding (GBS). The sliding interface located in the right bottom corner of the strain map is the one which resulted in cavitation (Fig. 6).
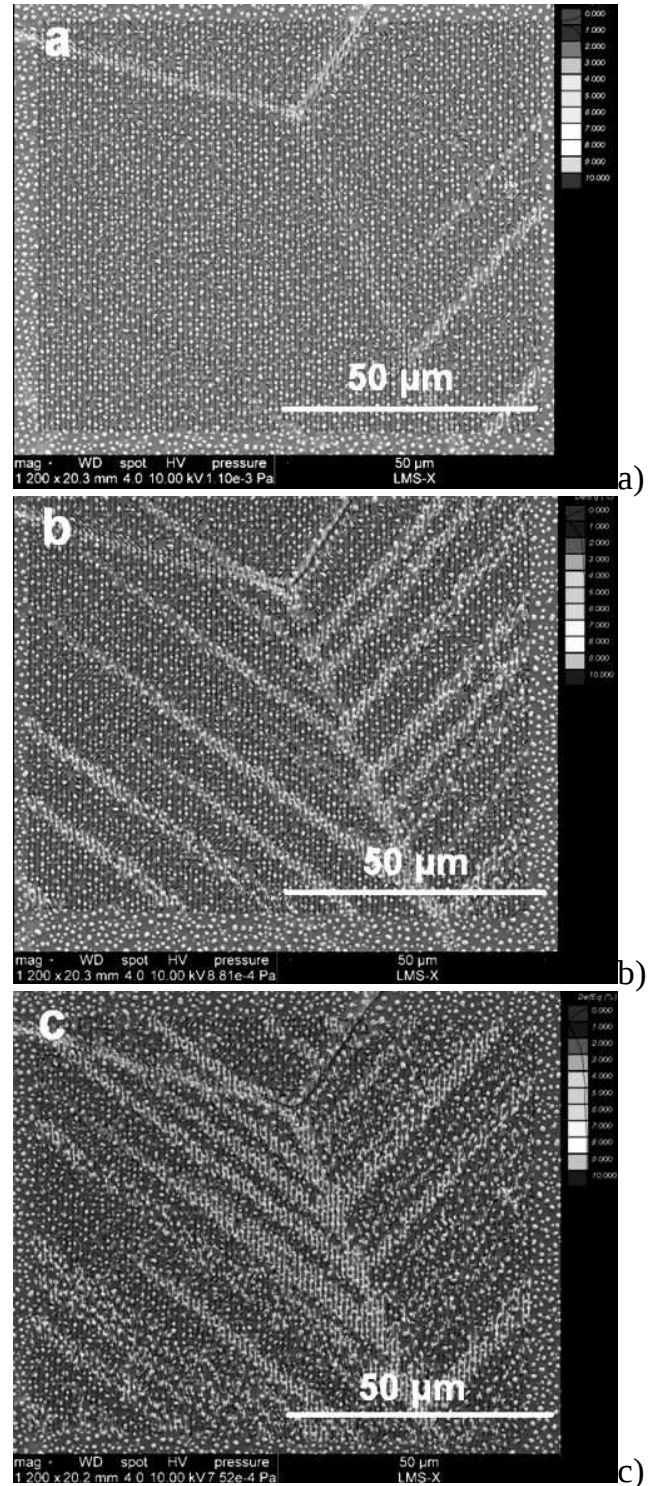

Figure 8 : Strain field obtained by DIC at the same steps of loading as in Fig. 7, but at the grain scale. The observed triple junction corresponds to the darker square area in the middle of Fig. 6.

Observations at the very local grain scale (Fig. 8) allowed us to highlight the interplays between the two different mechanisms of plastic deformation, namely crystal plasticity and grain sliding. It was for instance possible to follow chronologically the activation of each mechanism and its contribution to the local interactions between neighboring grains. Figure 8 shows in stage (a) essentially the activation of crystal plasticity within the right hand grain (and to a much lesser extent within the left hand one). Therefore, crystal plasticity incompatibility in between the right hand grain and the upper one (which is unable to activate easily crystalline slip) results in the beginning of GBS along their interface. With further loading, in stage (b) the GBS phenomenon increases, allowing for intensification of crystal plasticity in the left hand grain. At last, in stage (c) the deformation band activated within the latter grain is transmitted to the upper grain, which presents this time compatibility with respect to crys- 
tal plasticity. This triple point case illustrates the kind of interactions appearing between plastically anisotropic neighboring grains, which result in cooperation of crystal plasticity and grain boundary damage and sliding, and which finally condition the local heterogeneities of the strain field.

\section{DISCUSSIONS AND CONCLUSIONS}

For the uniaxially tested samples the loading curves and the identified dominant mechanism of crystal plasticity (Fig. 5 to 8), indicate viscoplastic behavior. The macroscopic conjugated diagonal shear bands, such as those observed in Figure 5, are a common feature, characteristic of the loading geometry. These shear bands are essentially ductile, but DIC demonstrated that they cumulate progressively dilation micro damage. The triaxially crept samples presented pronounced barreling (Fig. 9) and power law dependence of strain rate on differential stress, which characteristics indicate viscoplastic flow dominated by crystal plasticity. However, the observed stress exponent (of about 3) is lower than the value expected for crystal slip at room temperature conditions, which may indicate unsteady state conditions, or the activity of other mechanisms. The latter possibility is strengthened by the observation of substantial micro - damage, localized in numerous sets of conjugated shear bands within the barreled part of the specimens (Fig. 9). These observations are consistent with the findings of previous works (Peach and Spiers, 1996; Peach et al., 2001; Popp and Kern, 2001), showing that the room temperature flow of halite involved both ductile and brittle mechanisms: namely crystal plasticity and grain boundary micro-cracking. However, our work further demonstrated the cooperative nature of both mechanisms and highlighted their intimate relationships with local microstructure. We also provided quantitative estimations of the respective contribution of each mechanism at the experimental conditions. On the one hand, we have shown that crystal plasticity is the major strain accumulation mechanism (Fig. 6 to 8), accounting of more than $90 \%$ of the finite strain. But, we have also evidenced that local incompatibilities of crystal plasticity have induced interfacial micro - cracking and GBS (Fig. 10). At room temperature, the latter mechanisms in turn resulted in progressive accumulation of micro damage. The GBS mechanism accounted for less that $10 \%$ of the total finite strain, yet it was a necessary mechanism. Previous works have highlighted the importance of GBS as an accommodation mechanism in order to account for local strain incompatibilities related to the anisotropy of crystal plasticity
(Raj and Ashby, 1971; Dimanov et al., 2011; Raphanel et al., 2010). Such secondary, yet necessary mechanisms, allow for macroscopically homogeneous flow of anisotropic polycrystalline materials.

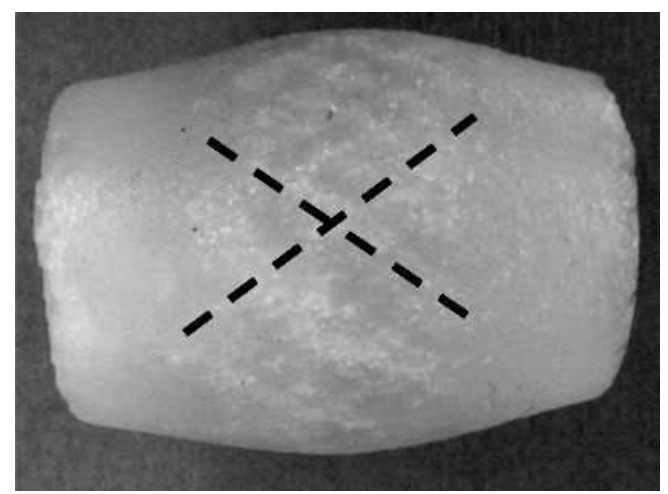

Figure 9 : Triaxially crept specimen (initially $2.5 \mathrm{~cm}$ in diameter) showing pronounced barreling (after $25 \%$ shortening) and numerous conjugated shear bands (indicated by the dashed lines) of cumulated damage in the central part.

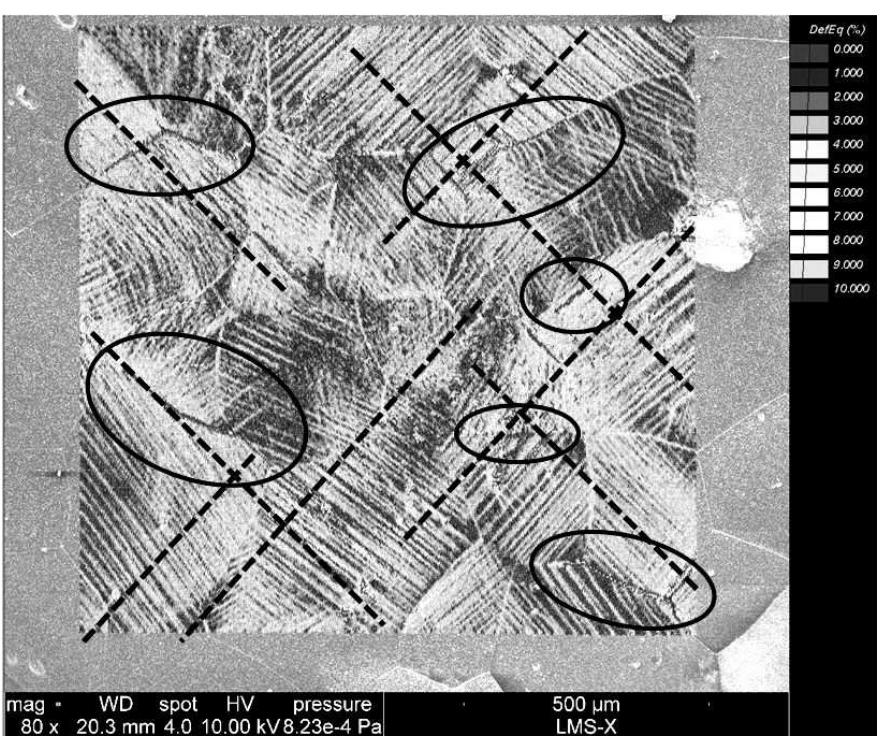

Figure 10 : Final deformation stage of the sample shown in Fig. 7. The individual grains show intracrystalline slip bands, which intensification and propagation throughout the microstructure forms mesoscopic shear bands (at $45^{\circ}$ to the principal stress). The circles indicate zones of identified grain boundary damage and sliding, in response to local incompatibilities of crystal plasticity.

It appears that at the experimental conditions crystal plasticity and GBS are coexisting and cooperative mechanisms (Fig. 10), and hence, that macroscopic viscoplastic flow and local interfacial damage are intimately linked. As a consequence, modeling the long term flow behavior of salt for the purpose of storage facilities solely on the basis of crystal plasticity (and corresponding experimentally derived flow laws) may be problematic, in the sense that its macroscopic viscoplastic behavior inherently involves a local brittle component, which may lead to permeability increases and premature failure, unless interfacial healing processes are involved. In 
any case, further micromechanical investigations are needed to better characterize and quantify the GBS phenomenon, depending on the specific loading and thermodynamic conditions, but also on the material microstructure itself.

\section{REFERENCES}

Dautriat, J., Bornert, M., Gland, N., Dimanov, A. and Raphanel, J. 2011. Localized deformation induced by heterogeneities in porous carbonate analysed by multi-scale digital image correlation. Tectonophysics., 503: 100-116.

Desbois G., Urai J.L., Kukla P.A., Konstanty J. and Baerle C. 2011a. High-resolution 3D fabric and porosity model in a tight gas sandstone reservoir: a new approach to investigate microstructures from mm- to nm-scale combining argon beam cross-sectioning and SEM imaging. J. Petrol. Sci. Eng., 78: 243-257.

Dimanov A., Raphanel J. and Dresen G. 2011. Newtonian flow of heterogeneous synthetic gabbros at high strain: Grain sliding, ductile failure, and contrasting local mechanisms and interactions. Eur. J. Mineral., 23(3): 303-322

Doumalin P., Bornert, M. and Caldemaison D. 1999. Microextensometry by DIC applied to micromechanical studies using SEM. Proc. Int. Conf. Adv. Techn. Exp. Mech. (Atem 99), 1 : 81-86.

Doumalin, P., Bornert M. and Crépin J. 2003. Caractérisation de la répartition de la déformation dans les matériaux hétérogènes. Mécanique et Industrie, 4 : 607-617.

Hickman, S. and Evans, B. 1995. Kinetics of pressure solution at halite-silica interfaces and intergranular clay films $J$. Geophys. Res., 100 (B7): 13113-1313.

Lebensohn, R.A., Dawson, P.R., Kern, H.M. and Wenk, H.R. 2003. Heterogeneous deformation and texture development in halite polycrystals: comparison of different modeling approaches and experimental data. Tectonophysics, 370 (1-4): 287-311.

Liu, Y., Gilormini, P. and Ponte Castañeda, P. (2005), Homogenization estimates for texture evolution in halite. Tectonophysics, Vol. 406 (3-4), 179-195.

Martin, B., Röller, K. and Stöckhert, B. (1999), Low-stress pressure solution experiments on halite single-crystals Tectonophysics, Vol. 308 (3), 299-310.

Peach, C.J. and Spiers, C.J. (1996). Influence of crystal plastic deformation on dilatancy and permeability development in synthetic salt rock. Tectonophysics, Vol. 256, 101-128.

Peach, C.J., Spiers, C.J. and Timby (2001). Effect of confiningp ressure on dilatation, recrystallization, and flow of rock salt at $150^{\circ}$ C. J. Geophys. Res., 106 (B3): 4061-4078.

Popp T. and Kern H. (2001). Evolution of dilatancy and permeability in rock salt during hydrostatic compaction and triaxiai deformation. J. Geophys. Res., 106 (B3): 4061-4078.

Raj R. \& Ashby, M.F. 1971. On grain boundary sliding and diffusional creep. Metallurgical Transactions, 2: 1113-1127.

Raphanel J., Dimanov A., Nazarova L.A., Nazarov, L.A. and Artemova A.I. 2010. High temperature rheology of synthetic two-phase gabbroic aggregates: microstructural heterogeneities and local deformation mechanism (2010). J. Mining Sci., 46(5): 495-502.

Urai, J.L., Spiers, C.J. Peach, C.J. Franssen, R.C.M.W. and Liezenberg, J.L. (1987), Deformation mechanisms operating in naturally deformed halite rocks as deduced from microstructural investigations. Geologie en Mijnbouw, 66: 165-176.

Wenk, H.R., G. Canova, A. Molinari and Mecking, H. (1989), Texture development in halite: Comparison of Taylor model and self-consistent theory. Acta Metall., 37(7): 2017-2029.

Wenk, H.R. Armann, M., Burlini, L., Kunze, K. and Bortolotti, M. (2009), Large strain shearing of halite: Experimental and theoretical evidence for dynamic texture changes. Earth and Planetary. Science Letters, 280 (1-4): 15205-15210. 